\title{
Zitrus-Sammlung im Palmengarten
}

\author{
Alexander Becker
}

\begin{abstract}
Since some years a Citrus collection is built up in the nursery of the Palmengarten. Some of the fruiting plants are shown in the flower conservatory near the nursery. It is designed like an orangery. History of orangeries and Citrus in Europe and Citrus in greek mythology are outlined.
\end{abstract}

\section{Zusamenfassung}

In der Gärtnerei des Palmengartens wird seit einigen Jahren eine Citrus-Sammlung aufgebaut. Einige der bereits fruchtenden Exemplare werden aktuell im Blütenhaus, das wie eine Orangerie gestaltet wurde, ausgestellt. Geschichte der Orangerien, Einführung von Citrus in Europa und Citrus in der griechischen Mythologie werden kurz vorgestellt.

\section{Zitrusfrüchte im Palmengarten}

Das Blütenhaus an der Gärtnerei zeigt seit vielen Jahren im jahreszeitlichen Wechsel kleine liebevoll gestaltete Blumenausstellungen. Diese sind mit Pflanzen, die entweder zu den festen Sammlungen der Gärtnerei gehören (z. B. Kamelien, Azaleen, Orchideen) oder speziell für Ausstellungszwecke in der Gärtnerei herangezogen werden (z. B. Zwiebelpflanzen, Weihnachtssterne) bestückt. Seit Herbst 2013 hat sich das Bild des Blütenhauses stark gewandelt. In mühevoller Handarbeit wurde im Kalthausbereich die Kulisse einer historischen Orangerie mit Säulen, Stuck und einer griechischen Götterstatue aufgebaut. Dazu werden hier nun verschiedene
Zitrus-Pflanzen ausgestellt, die Teil einer neuen, sich im Aufbau befindenden Zitrus-Sammlung sind. Bisher war die so wichtige und beliebte Nutzpflanzengattung Citrus im Palmengarten unterrepräsentiert und mit nur wenigen Exemplaren in einem der beiden Turmgewächshäuser seitlich des Hauses Leonhardsbrunn zu sehen.

\section{Entstehung der Sammlung}

Den Grundstock für die heutige Zitrus-Sammlung bildeten 20 Zitronen-, Limetten- und Pomeranzenbäumchen, welche für die Schmetterlingsausstellung 2005 als Futterpflanzen angeschafft worden waren. Nach der Ausstellung verschwanden die Zitruspflanzen ungenutzt

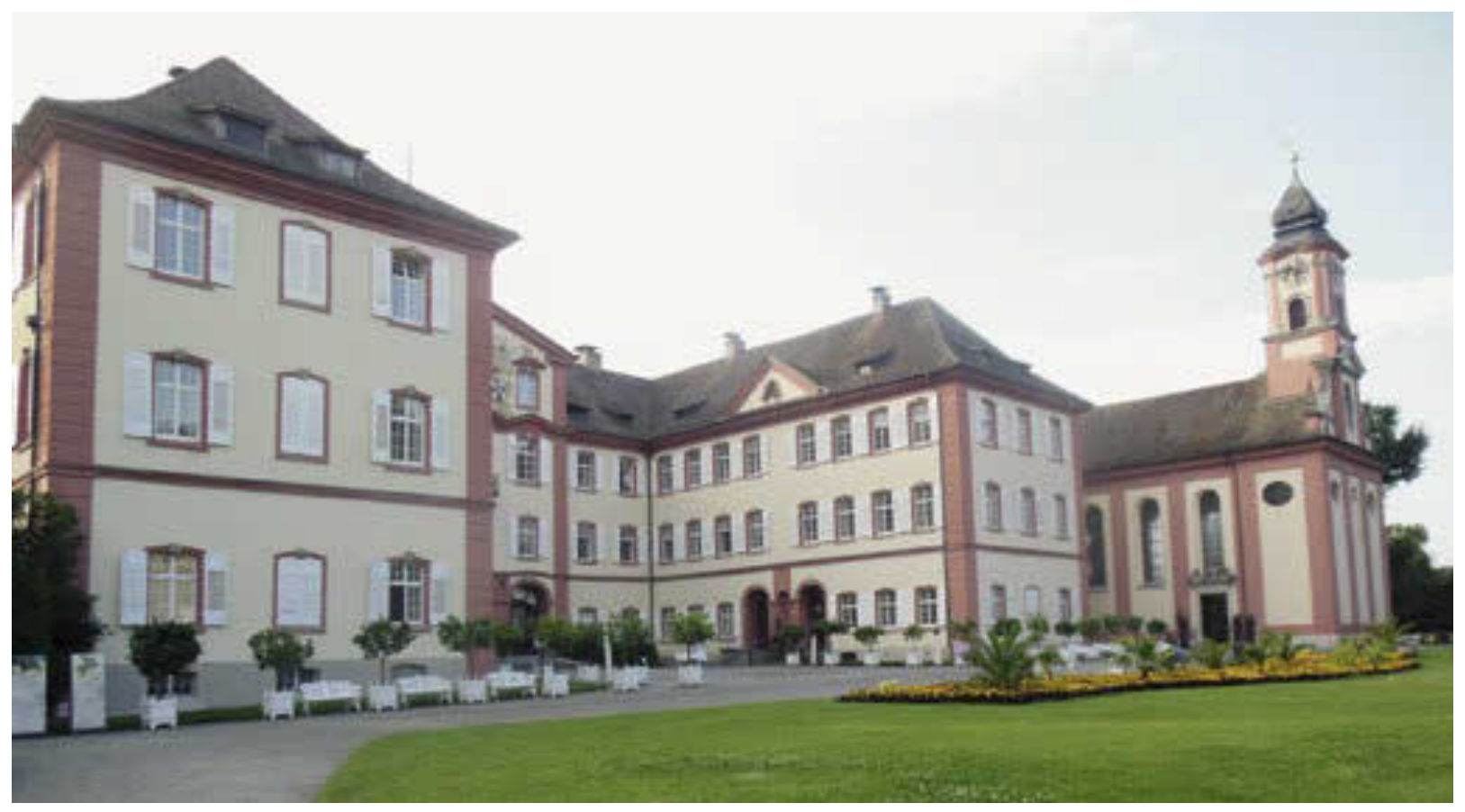


zwischen Ficus-Pflanzen. Im Jahr 2010 übernahm der Autor diesen Arbeitsbereich und kümmerte sich fortan intensiver um diese Kultur. Mit den vielen Versuchen von Düngung, Substraten und Vermehrungsweisen begann auch das Sammeln anderer Arten und Sorten. Durch die Unterstützung der „Freunde des Palmengartens e.V." konnte ein Teil ganz besonderer Sorten aus verschiedenen Gärtnereien zusammengetragen werden. Weitere Arten und Sorten wurden durch Veredlung selbst vermehrt. Die Edelreiser bekamen wir von anderen botanischen Gärten sowie Schloss- und Schaugärten. Auch der Erfahrungsaustausch mit den Mainau-Gärtnern war für den Autor sehr wertvoll und konnte bereits erfolgreich bei den Pflegearbeiten angewendet werden. Doch was nützt eine schöne Sammlung, wenn sie nur fern von den Besuchern hinter den Kulissen steht? Aus diesem Grund findet erstmals in diesem Jahr von September bis März eine kleine Präsentation der Zitrus-Pflanzen im Blütenhaus statt (s.o.). Durch die große Unterstützung unserer Betriebstechnik konnte der komplette Aufbau mit der Orangeriewand sowie Musikund Lichtinstallation schnell realisiert werden. Damit auch weiterhin die Zitrus-Pflanzen Verwendung finden, ist für 2019 eine große Zitrusausstellung geplant. Bis dahin müssen einige der noch sehr jungen und kleinen Pflanzen gepäppelt werden, sodass sie sich zu ansehnlichen Exemplaren entwickeln. Zusätzlich hat auch die Bildungseinrichtung „Kinder im Garten" die Zitrus-Pflanze in sein Programm genommen und hofft, dass auch unseren kleinen Besuchern die Pflanzen gefallen.

Abb. 1 (Seite 134): Historische Zitrus-Sammlung vor dem Schloss auf der Insel Mainau.

Abb. 2 (oben): Ein Teil der historischen Sorten der Insel Mainau. Die Pflanzen werden dort in Versailler Kübeln kultiviert.

Abb. 3 (Mitte): Orangerie im Schlosspark von Bad Homburg.

Abb. 4 (unten): Zitrus und andere Kübelpflanzen stehen zur Überwinterung in der Orangerie des Bad Homburger Schlosses.
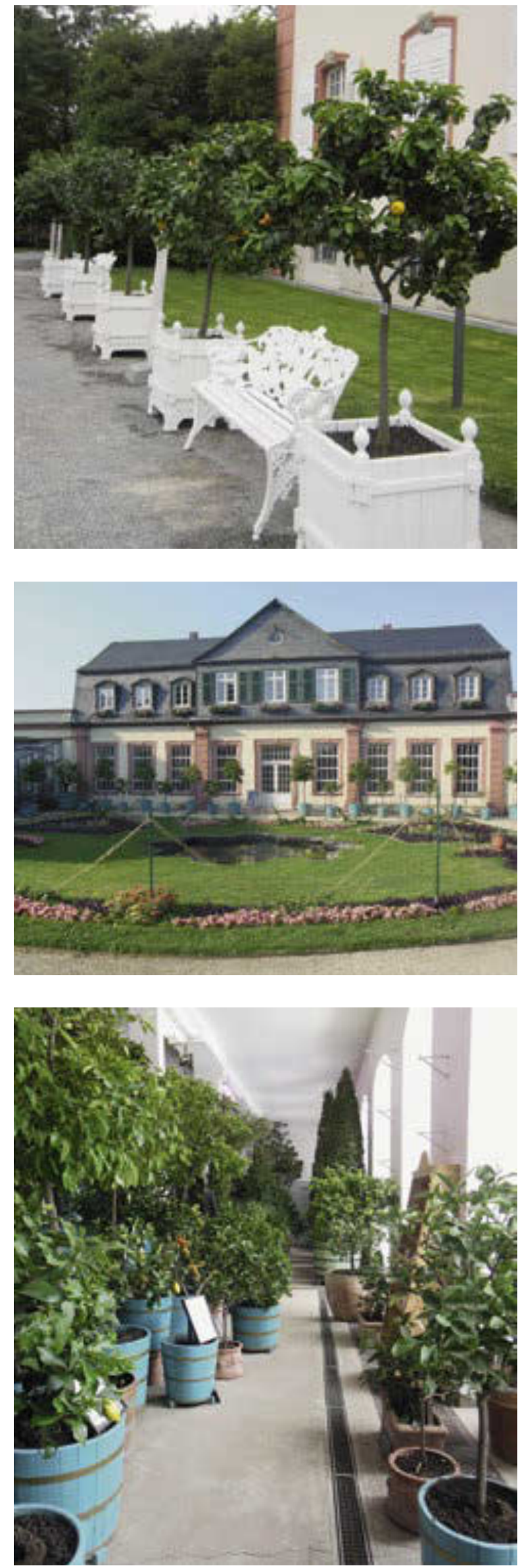

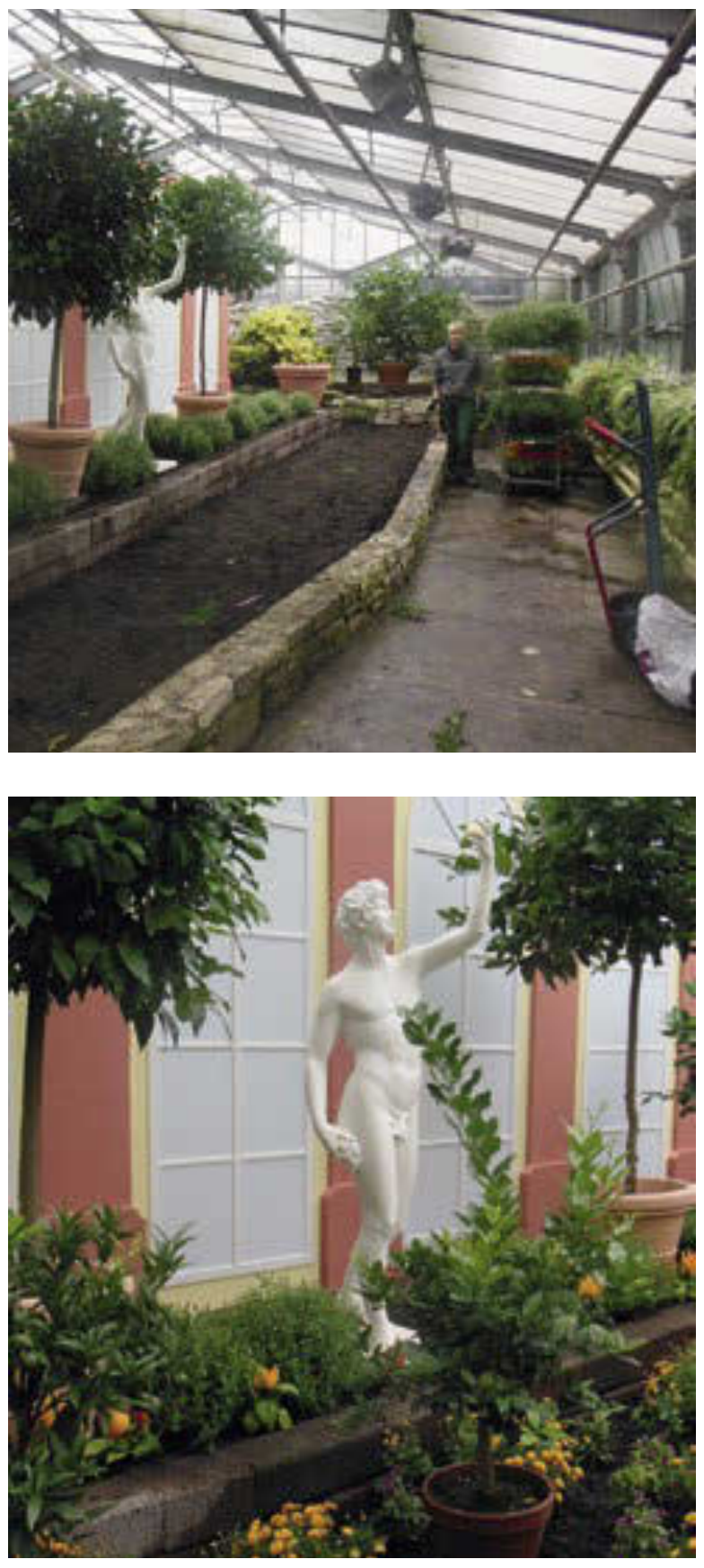

\section{Zitruspflanzen im Blütenhaus}

Das Blütenhaus zeigt bis zum Frühling einen Teil der Zitrus-Sammlung der Gärtnerei. Der Grund, sie gerade in den Wintermonaten zu zeigen, ist, dass die Früchte in dieser Zeit zu reifen beginnen und im Frühjahr die stark duftende Hauptblüte stattfindet. Da sich der größte Teil der Sammlung (ca. 90 verschiedene Arten und Sorten) noch im Aufbau befindet, werden nur die schon größeren und Früchte tragenden Pflanzen gezeigt.
Als Kulisse dient die Fassade einer Orangerie, um einen Eindruck zu vermitteln, wie es vom 17.-19. Jh. ausgesehen hat, wenn Pomeranzen, Zitronen und andere Zitrus-Bäume in den Sommermonaten vor den Orangerien aufgestellt wurden.

\section{Wie die Zitruspflanzen zu uns kamen}

Zitrus-Pflanzen zählen zu den ältesten Kulturgewächsen. In China wurden sie bereits vor 4000 Jahren in Kultur genommen. Erst um 330 v. Chr. wurde die Zitronat-Zitrone (Citrus medica) durch Feldzüge von Alexander dem Großen aus Persien nach Europa mitgebracht.

Ab dem 10. Jh. kamen Zitronen (Citrus limon) und Bitter-Orangen (Citrus aurantium) ins Mittelmeergebiet. Anfang des 16. Jh. schließlich wurden die ersten Süßorangen (Citrus sinensis) von Portugiesen über den Seeweg aus dem östlichen Asien mitgebracht. Der Anbau von Zitrusfrüchten nahm immer mehr zu, sodass der Mittelmeerraum heute mit eines der Hauptanbaugebiete ist (BAUMgARTNER et al. 2012).

\section{Orangerien}

Ab Mitte des 16. Jh. kamen Zitrus-Pflanzen über die Alpen auch zu uns und wurden an den Fürstenhöfen, aber auch bei reichen Bürgern, herangezogen und gepflegt. Es entstanden kleine Zitrus-Haine, überwiegend aus Pomeranzen. Um diese unbeschadet über den Winter zu bekommen, wurden sie im Herbst bis zu den ersten frostfreien Nächten mit barackenähnlichen, abschlagbaren „Pomeranzenhäusern“ überbaut. Diese waren aus Holz, besaßen kleine Fenster und wurden mit fahrbaren Öfen beheizt. Da der Auf- und Abbau ein enormer Aufwand war, wurden die Rück- und Seitenwände ab dem 17. Jh. zunehmend gemauert, sodass nur die Front und das Dach abschlagbar waren.

$\mathrm{Ab}$ dem 18. Jh. passten Pomeranzenhäuser und Haine aus ästhetischen Gründen nicht mehr in die neuen Barockgärten. Von nun an wurden die Pflanzen in transportablen Gefäßen und Kübeln kultiviert. Zur Überwinterung brachte man sie dann in feste Orangerie-Gebäude. Diese Orangerien waren nun Bestandteil vieler Schlösser und Gärten und wurden 
dementsprechend prachtvoll gebaut und verziert. In den Sommermonaten nutzte man die Orangerien häufig für Feste und Konzerte, was später schon beim Bau berücksichtigt wurde. Entsprechend wurden Überwinterungshallen teils auch mit Festsälen kombiniert. Ein gutes Beispiel ist der Dresdner Zwinger. Ursprünglich ließ August der Starke den Zwinger als Orangerie für etwa 4000 Zitrus-Pflanzen bauen. Später wurde der Zwinger dann immer mehr für Feste, Konzerte und Theateraufführungen genutzt. Durch solche Nutzungen konnten die Orangerien zu großen Gebäuden heranwachsen. Zum Beispiel hat die Orangerie in Potsdam eine Gesamtlänge von ca. $300 \mathrm{~m}$, in Wien Schönbrunn 189 m. Als im 19. Jh. die Barockgärten aus der Mode kamen und sich der englische Landschaftsgarten durchsetzte, verschwand das große Interesse an ZitrusPflanzen und Orangerien (Ahrendt et al. 2004, KLOCK \& KLOCK 2007).

\section{Die goldenen Äpfel der Hesperiden}

Für viele Fürstenhöfe war eine Zitrus-Sammlung ein Muss und Symbol für Tugend und Macht. Dieser Ruf gründete sich auf den Mythos vom Raub der goldenen Äpfel der Hesperiden:

Nach griechischer Mythologie schenkte die Erdmutter Gaia Zeus und Hera zur Hochzeit einen Baum mit goldenen Äpfeln. Dieser wurde in den Garten der Götter gepflanzt und von den Hesperiden, den Töchtern des Atlas und der Nacht, gepflegt und durch eine doppelköpfige Schlange oder einen Drachen namens LAdON gut bewacht. Eurystheus stellte HerKULES zwölf Aufgaben, von denen die elfte der Raub dieser goldenen Äpfel war. Nach einer Überlieferung überwältigte Herkules die Schlange Ladon und konnte so die Äpfel aus dem Garten stehlen. Nach einer anderen Fassung musste Herkules eine List anwenden. Er ließ Atlas die Äpfel stehlen und nahm ihm

Abb. 5 (Seite 136 oben): Neugestaltung des Blütenhauses. Abb. 6 (Seite 136 Mitte): Zitrus im Blütenhaus.

Abb. 7 (oben): Eine Frucht der Sorte 'Buddhas Hand'.

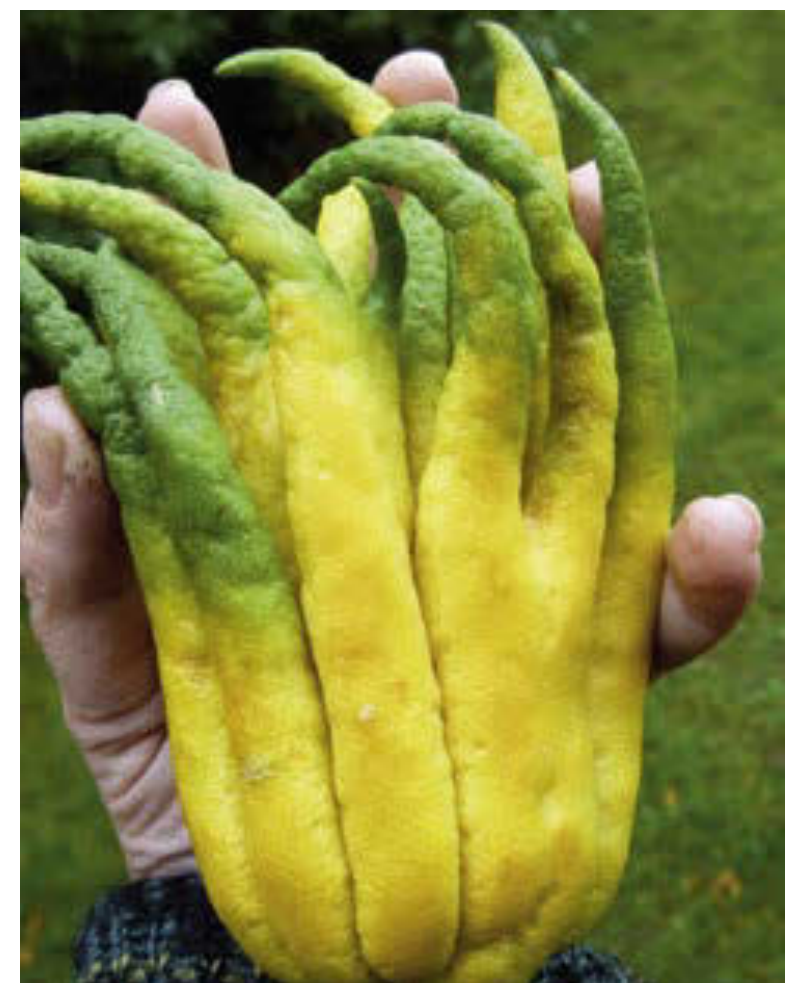

dafür die Last des Himmelgewölbes ab, die Atlas zur Strafe für die Teilnahme an einem Kampf gegen Zeus tragen musste. Als dieser nun mit den Äpfeln zurückkehrte und diese selbst Eurystheus bringen wollte, bat ihn Herkules, ihm das Gewölbe nochmal kurz abzunehmen, damit er sich ein Kissen auf die Schultern legen könne. ATLas willigte ein und nahm ihm die Last ab. So konnte Herkules die goldenen Äpfel zu Eurystheus bringen. Nachdem damit die Aufgabe gelöst war, ließ Eurystheus die goldenen Äpfel wieder zurück in den Garten der Hesperiden bringen.

\footnotetext{
Literatur

Ahrendt, D., Balsam, S., Elfgang, A., Grimm, U., Gröschel, C., Hamann, H., Münzenmayer, R., Proske, H.-D., Wertz, H. W. 2004: Der Süden im Norden - Orangerien, ein fürstliches Vergnügen. - Stuttgart.
}

Baumgartner, T., Dietrich, G., Fischer-Colbrie, P., Gröschel, C., Kalous, E., Karner, H., Klock, P., Reitbauer, H., Wiegele, M. \& Thun-Täubert, E. 2012: Über Orangen und Zitronen. - Schriftreihe der Österreichischen Gartenbau-Gesellschaft 1. - Wien.

KLOCK, M. \& KLOCK, T. 2007: Das große Ulmer-Buch der Zitruspflanzen. - Stuttgart. 